\title{
Comparison of Acute Bout of Moderate Intensity and High Intensity Exercise on Oxidative Stress, Inflammatory Markers and Neurocognitive Parameters between Male Athletes and Non-athletes - A Randomized Controlled Study
}

\author{
Keni Gowsi ${ }^{1, *}$, Gopal Krushna Pal' ${ }^{1}$ Karthick Subramanian ${ }^{2}$
}

\section{Keni Gowsi ${ }^{1}, *$, Gopal Krushna Pal' ${ }^{1}$, Karthick Subramanian ${ }^{2}$}

'Department of Physiology, Jawaharlal Institute of Post Graduate Medical

Education and Research, Puducherry, INDIA.

${ }^{2}$ Department of Psychiatry, Mahatma Gandhi Medical College and Research Institute, Sri Balaji Vidyapeeth,

Puducherry, INDIA.

\section{${ }^{*}$ Correspondence}

\section{Dr. Keni Gowsi}

Senior Resident, Department of Physiology, Jawaharlal Institute of Post Graduate Medical Education and Research, Puducherry-605 006, INDIA.

Phone: +91-9626610599

Email:keni_doc@yahoo.in

\section{History}

- Submission Date: 05-08-2019;

- Review completed: 09-10-2019;

- Accepted Date: 16-11-2019.

DOI : 10.5530/ijcep.2019.6.4.36

\section{Copyright}

(C) 2019 Phcog.Net. This is an openaccess article distributed under the terms of the Creative Commons Attribution 4.0 International license.

\begin{abstract}
Background and Aim: Physical inactivity and resultant obesity are highly prevalent in the contemporary world. Studies reveal that even an acute bout of physical exercise can bring about health benefits similar to that of regular exercise. Our study aims to assess the effect of single acute bout of exercise on cognition, oxidative stress and inflammatory markers between athletes and non-athletes and to compare the effect of moderate and high intensity exercise on those parameters. Methods: The study is a double-arm parallel design randomized controlled study. Eighty healthy male volunteers (40 athletes and 40 non-athletes) were recruited and further randomized into four subgroups: Athlete moderate-intensity exercise, athlete high-intensity exercise, non-athlete moderate-intensity exercise and non-athlete high-intensity exercise. A single acute bout of treadmill exercise was given to all the subgroups. Cognitive parameters, oxidative stress and inflammatory markers were assessed before and after acute bout of exercise and compared between the groups. Results: At baseline, athletes had reduced oxidative stress and inflammation than non-athletes, which is evident by increased total anti-oxidant status and decreased leptin in athletes, whereas no significant changes in cognitive parameters between the groups. In addition, we found that moderate intensity exercise is superior to high intensity exercise in decreasing inflammation and oxidative stress in both the groups. However, no such changes were observed with respect to cognitive parameters with different intensities of exercise. Conclusion: $A$ single acute bout of exercise is sufficient to improve oxidative and inflammatory parameters in both athletes and non-athletes, whereas cognitive functions does not improve by single acute bout of exercise.

Key words: Acute exercise, Athlete, Non-athlete, Antioxidant status, Inflammatory markers, Cognition, p300 latency.
\end{abstract}

\section{INTRODUCTION}

Physical inactivity and sedentary lifestyle are the major risk factors for most of the non-communicable diseases such as obesity, ${ }^{[1]}$ type 2 diabetes mellitus, ${ }^{[2]}$ hypertension, ${ }^{[3]}$ coronary artery disease, ${ }^{[4]}$ and many others. It has been reported that, worldwide $31.1 \%$ of the adults are physically inactive. ${ }^{[5]}$

Sedentary lifestyle leads to obesity, which initiates the degeneration processes resulting in oxidative stress and chronic inflammation. ${ }^{[6]}$ Oxidative stress reflects an imbalance between production of reactive oxygen species and antioxidant defence mechanisms ${ }^{[7]}$ The alterations of the normal redox state of cells causes cellular and tissue damage leading to aging, inflammatory, cardiovascular, neurodegenerative diseases and cancers. ${ }^{[8]}$ On the contrary, physical activity is inversely associated with oxidative stress ${ }^{[7]}$

and low-grade inflammation by means of exerciseinduced redox adaptations. ${ }^{[9,10]}$

Apart from its health benefits, physical activity has a positive impact on cognition. ${ }^{[11]}$ Several human and animal studies have revealed the neurobiological mechanisms underlying the impact of physical activity on cognition. ${ }^{[12]}$ Studies have documented that aerobic exercise is associated with positive neurological and cognitive outcomes in children and older adults. ${ }^{[13]}$ Previous studies have shown that not only chronic exercise, even acute physical exercise may improve alertness, processing speed and executive functions of cognition. ${ }^{[14]}$

Exercise enhances executive aspects of cognition, which includes inhibition, working memory and cognitive flexibility. ${ }^{[13]}$ While some studies reveal improvement in cognition with both moderate and

Cite this article: Gowsi K, Pal GK, Subramanian K. Comparison of Acute Bout of Moderate Intensity and High Intensity Exercise on Oxidative Stress, Inflammatory Markers and Neurocognitive Parameters between Male Athletes and Non-athletes - A Randomized Controlled Study. Int J Clin Exp Physiol. 2019;6(4):139-43. 
high intensity exercises, contrary findings do exists which reveal that high intensity exercise may not have beneficial effects on cognition, rather worsen it and moderate intensity exercises are more beneficial. ${ }^{[15]}$

There is a paucity in the literature regarding the effect of single acute bout of exercise on oxidative stress, inflammation and cognition. The present study aimed to elicit the impact of acute bout of exercise on oxidative stress, inflammation and cognitive functions in athletes and non-athletes. We also aimed to explore the effects of various intensities (moderate and high intensities) of exercise on the above said parameters in athletes and non-athletes.

\section{METHODS}

\section{Setting}

The present study was conducted in a tertiary hospital cum research institute of southern India, JIPMER, Puducherry. The study participants reported to the Cardiovascular Research Lab in the Department of Physiology. The lab is equipped with a treadmill, blood pressure, heart rate and Electrocardiograph (ECG) monitor, a couch for pre and postexercise recordings and a computerised software (Cardiosoft) for selecting the protocol for treadmill test. The room temperature was maintained between $25^{\circ} \mathrm{C}$ to $27^{\circ} \mathrm{C}$.

\section{Participants}

Eighty healthy male volunteers (40 athletes and 40 non-athletes) in the age group of 18-25 years participated in the study. The athletes were recruited from the Department of Physical Education and Sports, School of Humanities, Pondicherry University. The selection criteria for athletes included the following: the athletes should have undergone supervised structured physical conditioning training for at least past one year and should have participated in a regional/national/international sports athletic event. For our study, we selected different category of players including football players, hockey players, runners etc., on a random basis. Age and BMI-matched male non-athletes who had not participated in the athletic/sports events and not underwent any structured physical conditioning training in the past year were concurrently recruited for the study. The study participants were administered "International Physical Activity Questionnaire (IPAQ)" before performing the treadmill test. The IPAQ is an objective method to assess the physical activity levels of the participants.

Subjects with chronic medical illnesses (inflammatory conditions, diabetes, hypertension, cardiovascular, respiratory or neurological disorders), comorbid severe cardiac or pulmonary illnesses which prevented them from performing treadmill test, were excluded from the study.

\section{Study Design}

The study was conducted as a double-arm parallel-group randomized controlled trial (RCT). The study included 80 participants, out of which 40 were athletes and 40 were non-athletes. These two groups were further randomized into two sub-groups; therefore, there were four subgroups: moderate-intensity athlete, high-intensity athlete, moderateintensity non-athlete, high-intensity non-athlete. The subgroups allocation was based on the computer-generated block randomization method. The sample size was 80 , with 40 in each group and 20 in each subgroup.

\section{Study Procedure}

The Institute scientific advisory committee and human ethics committee approved the study. A written informed consent was obtained from all the participants. The subjects were instructed to avoid drinking beverages and performing vigorous exercises on the day of the procedure. They reported to the Cardiovascular Research Lab in the Department of
Physiology, in fasting state at 8 am on the day of procedure. $5 \mathrm{ml}$ of venous blood was drawn to assess the baseline levels of the inflammatory markers and total anti-oxidant status. The participants were given a light meal of equivalent calories and were allowed a brief (30 $\mathrm{min}$ ) resting period. Following which, the baseline anthropometric and cognitive parameters including p300, Visual Reaction Time (VRT) and Auditory Reaction Time (ART) were recorded. P300 is the Event Related Potential (ERP), which was recorded using "Neuropack MEB 9200/9300 vs. 08-15" software in a soundproof lab.

Based on the randomization method, the athletes and non-athletes were further divided into sub-groups. All the 80 participants underwent single acute bout of exercise in the computerised motor driven treadmill. Of which, two sub-groups (includes athletes and non-athletes) performed moderate-intensity exercise and the other two sub-groups performed high-intensity exercise in the treadmill based on the Bruce Ramp Protocol. This protocol is widely used to assess the cardiorespiratory fitness, consisting of multiple stages with fixed grade and speed along each progressive stage.

The advantages of using a Bruce Ramp Protocol (BSU) over the standard Bruce protocol is, the improved ability of BSU protocol to predict maximal oxygen uptake $\left(\mathrm{VO}_{2}\right.$ max), acceptable exercise test time, more uniform gas exchange and hemodynamic responses during the exercise test. A fundamental feature of this protocol is that physical work settings on the treadmill (i.e., speed and grade) are equivalent at each 3-min period to the standard Bruce protocol. All these advantages make the BSU/Bruce Ramp protocol an excellent choice for laboratories desiring to calculate the $\mathrm{VO}_{2}$ max using treadmill. ${ }^{[16]}$

Heart rate, blood pressure and ECG were continuously monitored throughout the exercise and recovery period. Blood pressure was monitored by "Suntech Tango," in which ECG signal and the Korotkoff sounds were collected by the microphone in the cuff and transmitted to the software. ECG monitoring was done with the help of 12-lead ECG. The heart rate of the subjects were maintained between $64 \%$ to $77 \%$ of maximum heart rate $\left(\mathrm{HR}_{\max }\right)$ in moderate intensity exercise and $77 \%$ to 94\% in high-intensity exercise. This $\mathrm{HR}_{\max }$ was calculated from the standardized formula 220-Age.

Borg's rating of perceived exertion scale was used throughout the treadmill test to assess the exertion level of the subject during the test. Once the subject reaches the target heart rate, exercise was stopped and recovery period of ten minutes was given, during which heart rate, blood pressure and ECG were continuously monitored.

After heart rate recovery, five $\mathrm{ml}$ of venous blood was withdrawn to assess the post-exercise levels of inflammatory and oxidative stress markers and post-exercise levels of p300, VRT and ART were evaluated. The blood samples were centrifuged and stored at $-80^{\circ} \mathrm{C}$. The inflammatory markers and total anti-oxidant status were quantitatively assessed using ELISA technique.

\section{Statistical Analysis of Data}

Data were entered and analysed using SPSS 19.0. Paired $t$ test was used to compare means before and after exercise. Independent $t$ test was used to compare means across the groups in normal distribution. One-way ANOVA was used to compare the means of the subgroups (athletes and non-athletes of moderate and high intensity) of normal distribution. A $p$-value of $<0.05$ was considered as statistically significant.

\section{RESULTS}

Table 1 summarizes the anthropometric parameters of the study and the control group. No significant differences existed in the anthropometric parameters between the groups (Table 1). 
Table 2 summarizes the effect of intervention (single acute bout of exercise) on oxidative stress and inflammatory markers in all the four subgroups. The baseline and post-exercise values of athletes of moderate and high intensity were compared with non-athletes of moderate and high intensity.

We found a significant difference in baseline values of total antioxidant and leptin between athletes and non-athletes. Athletes had higher values of total anti-oxidant and lower levels of leptin (pro-inflammatory marker) when compared with non-athletes at baseline. Total adiponectin (antiinflammatory marker) did not differ much between athletes and nonathletes. When we compare moderate with high intensity exercise, we found a statistically significant improvement with moderate intensity exercise in both athletes and non-athletes in total antioxidant and leptin values. Athletes showed a dramatic improvement with moderate intensity exercise than non-athletes.

Table 3 summarizes the effect of intervention (single acute bout of exercise) on cognitive parameters in all the four subgroups.

\section{DISCUSSION}

Our results demonstrate that inflammation and oxidative stress are lower in athletes when compared to non-athletes at baseline. These findings are in line with previous studies, which states that regular exercise makes individuals stronger against oxidative stress, increases the activity of antioxidant defence system and reduces oxidant production. ${ }^{[17-19]}$ Even though both athletes and non-athletes showed better results with

Table 1: Baseline characteristics of athletes $(n=40)$ and non-athletes $(n=40)$.

\begin{tabular}{|cccc|}
\hline Parameter & Athletes & Non-athletes & $P$-value \\
\hline Age (in years) & $23.45 \pm 1.67$ & $23.60 \pm 1.34$ & 0.658 \\
Height (in cm) & $169.04 \pm 4.78$ & $170.69 \pm 6.25$ & 0.188 \\
Weight (in Kg) & $67.11 \pm 5.99$ & $70.24 \pm 14.25$ & 0.205 \\
BMI $\left(\mathrm{Kg} / \mathrm{m}^{2}\right)$ & $23.49 \pm 2.01$ & $24.09 \pm 4.13$ & 0.414 \\
\hline
\end{tabular}

Data are expressed as Mean \pm SD. The baseline values of athletes and non-athletes were compared using independent student's ' $t$ ' test. ${ }^{*} P<0.05$ has been considered as statistically significant. BMI-Body Mass Index moderate intensity exercise, athletes showed a dramatic improvement than non-athletes. Regular exercise also protects against diseases associated with chronic low-grade systemic inflammation. This long-term effect of exercise may be ascribed to the reduction in the pro-inflammatory marker leptin, with single bout of exercise. ${ }^{[20]}$

In sedentary individuals, the concentration of the protective enzymes (against free radicals) such as superoxide dismutase and glutathione peroxidase, are reduced. ${ }^{[21]}$ In addition, a previous study has demonstrated a decrease in the non-enzymatic system, particularly ascorbic acid concentration. ${ }^{[22]}$ These reductions in the protective enzymatic and non-enzymatic system against free radicals results in increased oxidative stress in physically inactive individuals. In addition, previous studies states that increasing amounts of sedentary behaviour were associated with unfavourable alterations in the inflammatory profile, which increases the inflammation. ${ }^{[23]}$

When we compare moderate with high intensity exercise, we found a significant improvement in total antioxidant and leptin values with moderate intensity exercise in both athletes and non-athletes. Our findings are supported by the studies, which states that moderate intensity exercise inhibits free radical generation and increases the antioxidant levels ${ }^{[24]}$ and inflammation, while high intensity exercise induces the free radical generation. ${ }^{[25]}$ However, there is a difference of opinion in the literature while some studies reports that high intensity exercise attenuates oxidative stress than moderate intensity exercise. ${ }^{[26]}$ Whereas, others reason out that both moderate and high intensity exercises were beneficial. ${ }^{[27,28]}$

Unlike inflammatory and oxidative stress parameters, which improve with single acute bout of exercise, cognition does not improve with acute bouts of moderate intensity or high intensity exercise. The reason is that regular exercise improves cognition by increasing the number of cortical capillaries, elevating the Brain-derived Neurotrophic Factor (BDNF) levels, stimulating the neurogenesis and synaptic plasticity, eventually culminating in enhanced learning and cognitive functions. ${ }^{[29,30]}$ To produce all the above said changes, the subject has to undergo regular physical activity.

Our results are supported by previous studies, which reports that physical fitness, but not an acute bout of aerobic exercise enhances cognitive processing. ${ }^{[31]}$ Another study has reported that acute exercise has short-term

Table 2: Comparison of oxidative stress and inflammatory markers between athletes and non-athletes of moderate and high intensity exercises.

\begin{tabular}{|c|c|c|c|c|c|}
\hline \multicolumn{2}{|c|}{ Parameter } & \multicolumn{2}{|c|}{ Athletes } & \multicolumn{2}{|c|}{ Non-Athletes } \\
\hline & & Moderate Intensity & High Intensity & Moderate Intensity & High Intensity \\
\hline \multirow{2}{*}{ Adiponectin } & Pre-exercise & $15.57 \pm 6.88$ & $18.24 \pm 7.87$ & $15.48 \pm 7.23$ & $16.11 \pm 7.07$ \\
\hline & Post exercise & $17.80 \pm 7.65$ & $18.23 \pm 6.80$ & $16.80 \pm 7.51$ & $17.24 \pm 9.08$ \\
\hline \multirow{2}{*}{ Total Antioxidant } & Pre-exercise & $37.58 \pm 10.94$ & $27.71^{\star} \pm 8.40$ & $9.62^{* * * *} \pm 1.88$ & $7.78^{* * * \#} \pm 2.57$ \\
\hline & Post exercise & $38.43 \pm 8.27$ & $27.73^{\star} \pm 7.41$ & $10.66^{* * * *} \pm 1.22$ & $8.77^{\star * * \#} \pm 2.51$ \\
\hline \multirow{2}{*}{ Leptin } & Pre-exercise & $2.12 \pm 1.47$ & $3.79^{*} \pm 0.51$ & $5.86^{* \star} \pm 1.17$ & $9.25^{\#} \pm 2.39$ \\
\hline & Post exercise & $1.53 \pm 0.7$ & $3.29^{*} \pm 0.58$ & $5.40^{* * *} \pm 1.01$ & $7.82^{\# \#} \pm 1.98$ \\
\hline
\end{tabular}

Data are expressed as Mean \pm SD. One-way ANOVA was used to compare the means of the subgroups (athletes and non-athletes of moderate and high intensity) of normal distribution. A $p$-value of $<0.05$ was considered as statistically significant

The ${ }^{\star}$ mark indicates comparison of data of subgroups (high intensity athletes, moderate intensity non-athletes, high intensity non-athletes) with moderate intensity athletes.

The * mark indicates comparison of data of subgroups (moderate intensity athletes, moderate intensity non-athletes, high intensity non-athletes) with high intensity athletes.

${ }^{*} P<0.05,{ }^{* *} P<0.01,{ }^{* * *} P<0.001$

$\# P<0.05$

$\# P<0.01$ 
Gowsi, et al.: Acute Exercise on Inflammatory Markers, Oxidative Stress and Neurocognitive Parameters

Table 3: Comparison of cognitive parameters between athletes and non-athletes of moderate and high intensity exercises.

\begin{tabular}{|c|c|c|c|c|c|c|c|}
\hline \multirow{2}{*}{ Parameter } & & \multicolumn{2}{|c|}{ Athletes } & \multicolumn{2}{|c|}{ Non-Athletes } & \multirow[b]{2}{*}{ F statistic } & \multirow[b]{2}{*}{ P-value } \\
\hline & & Moderate Intensity & High Intensity & Moderate Intensity & High Intensity & & \\
\hline P300 Latency & Pre-exercise & $351.1(19.85)$ & $352.3(24.25)$ & $351.1(26.43)$ & 355.9 (29.57) & 0.162 & 0.922 \\
\hline \multirow{2}{*}{ Visual Reaction Time } & Pre-exercise & $174.75(10.94)$ & $171.65(10.64)$ & $172(18.7)$ & $174.85(17.41)$ & 1.467 & 0.230 \\
\hline & Post exercise & $152.8(14.9)$ & $156.95(17.53)$ & $153.25(13.97)$ & $154.80(15.38)$ & 0.090 & 0.965 \\
\hline
\end{tabular}

Data are expressed as Mean \pm SD. One-way ANOVA was used to compare the means of the subgroups (athletes and non-athletes of moderate and high intensity) of normal distribution. A $p$-value of $<0.05$ was considered as statistically significant

benefits for processing speed but does not affect other types of cognitive functioning. ${ }^{[32,33]}$ Therefore, it is evident that acute exercise does not enhance neurogenesis and synaptogenesis significantly. In contrast to our studies, few articles have reported that single bout of exercise benefits cognitive processes by improving executive functions and processing speed. ${ }^{[34,35]}$

\section{CONCLUSION}

To conclude, our study results shows that athletes had lower oxidative stress and inflammation in baseline and showed better improvement following intervention with single acute bout of moderate intensity exercise than non-athletes. When we compared moderate with high intensity exercise, we found out that moderate intensity exercise is superior to high intensity exercise in producing significant changes in inflammatory and anti-oxidant parameters in both athletes and non-athletes. We also found that, unlike oxidative and inflammatory markers, cognition does not improve with single acute bout of exercise in both the groups. Future studies with a larger sample size are needed to replicate such findings and explore the influence of exercise intensity on neurocognitive functions.

\section{ACKNOWLEDGEMENT}

The authors thank the study subjects for their participation in the study.

\section{CONFLICT OF INTEREST}

The authors declare no conflict of interest.

\section{Source of Funding}

The study was funded through the intramural funding agency of the institute.

\section{ABBREVIATIONS}

IPAQ: International Physical Activity Questionnaire; ECG: Electrocardiograph; VRT: Visual Reaction Time; ART: Auditory Reaction Time; ERP: Event Related Potential; RCT: Randomized Controlled Trial; BSU: Bruce Ramp Protocol; $\mathbf{V}_{\mathrm{O} 2}$ max: Maximal Oxygen Uptake; $\mathbf{H R}_{\max }$ : Maximum Heart Rate; BDNF: Brain-derived Neurotrophic Factor

\section{REFERENCES}

1. Vincent HK, Innes KE, Vincent KR. Oxidative stress and potential interventions to reduce oxidative stress in overweight and obesity. Dia Obes Metab. 2007; 9(6):813-39

2. Larsen BA, Martin L, Strong DR. Sedentary behavior and prevalent diabetes in non-Latino whites, non-Latino blacks and Latinos: Findings from the National Health Interview Survey. J Pub Health. 2014;37(4):634-40.

3. Beunza JJ, Martínez-González MÁ, Ebrahim S, Bes-Rastrollo M, Núnez J,
Martínez JA et al. Sedentary behaviors and the risk of incident hypertension: The SUN Cohort. Am J Hyperten. 2007;20(11):1156-62.

4. Hamer M, Venuraju SM, Urbanova L, Lahiri A, Steptoe A. Physical activity, sedentary time and pericardial fat in healthy older adults. Obesity. 2012;20(10):2113-7.

5. Hallal PC, Andersen LB, Bull FC, Guthold R, Haskell W, Ekelund U, et al. Global physical activity levels: Surveillance progress, pitfalls and prospects. The Lancet. 2012;380(9838):247-57.

6. Vincent HK, Morgan JW, Vincent KR. Obesity exacerbates oxidative stress levels after acute exercise. Med Sci Sports Exerc. 2004;36(5):772-9.

7. Carraro E, Schilirò T, Biorci F, Romanazzi V, Degan R, Buonocore D, et al. Physical activity, lifestyle factors and oxidative stress in middle age healthy subjects. Int J Environ Res Public Health. 2018;15(6):1152.

8. Pingitore A, Lima GPP, Mastorci F, Quinones A, lervasi G, Vassalle C. Exercise and oxidative stress: Potential effects of antioxidant dietary strategies in sports. Nutrition. 2015;31(7-8):916-22.

9. Geffken DF, Cushman M, Burke GL, Polak JF, Sakkinen PA, Tracy RP. Association between physical activity and markers of inflammation in a healthy elderly population. Am J Epidemiol. 2001;153(3):242-50.

10. Menicucci D, Piarulli A, Mastorci F, Sebastiani L, Laurino M, Garbella E, et al. Interactions between immune, stress-related hormonal and cardiovascular systems following strenuous physical exercise. Archi Italie Biol. 2013;151(3):126-36.

11. Herting MM, Chu X. Exercise, cognition and the adolescent brain. Birt Defec Res. 2017;109(20):1672-9.

12. Yanagisawa H, Dan I, Tsuzuki D, Kato M, Okamoto M, Kyutoku Y, et al. Acute moderate exercise elicits increased dorsolateral prefrontal activation and improves cognitive performance with Stroop test. Neuroimage. 2010;50(4):1702-10.

13. Hillman $\mathrm{CH}$, Pontifex MB, Raine LB, Castelli DM, Hall EE, Kramer AF. The effect of acute treadmill walking on cognitive control and academic achievement in preadolescent children. Neuroscience. 2009;159(3):1044-54.

14. Córdova C, Silva VC, Moraes CF, Simões HG, Nóbrega ODT. Acute exercise performed close to the anaerobic threshold improves cognitive performance in elderly females. Braz J Med Biol Res. 2009;42(5):458-64.

15. Stanford KI, Goodyear LJ. Exercise and type 2 diabetes: Molecular mechanisms regulating glucose uptake in skeletal muscle. Adv Physiol Edu. 2014;38(4):308-14

16. Kaminsky LA, Whaley MH. Evaluation of a new standardized ramp protocol: The BSU/Bruce Ramp protocol. J Cardiopulm Rehab Prev. 1998;18(6):438-44.

17. Djordjevic DZ, Cubrilo DG, Barudzic NS, Vuletic MS, Zivkovic VI, Nesic M, et al. Comparison of blood pro/antioxidant levels before and after acute exercise in athletes and non-athletes. Gen Physiol Biophys. 2012;31(2):211-9.

18. Marin DP, Bolin AP, Campoio TR, Guerra BA, Otton R. Oxidative stress and antioxidant status response of handball athletes: Implications for sport training monitoring. Int Immunopharmacol. 2013;17(2):462-70.

19. Sharifi G, Najafabadi AB, Ghashghaei FE. Oxidative stress and total antioxidant capacity in handball players. Adv Biomed Res. 2014;3.

20. Petersen AMW, Pedersen BK. The anti-inflammatory effect of exercise. J Appl Physiol. 2005;98(4):1154-62.

21. Marciniak A, Brzeszczynska J, Gwozdzinski K, Jegier A. Antioxidant Capacity and Physical Exercise. Biol Sport. 2009;26(3).

22. Jammes Y, Steinberg JG, Brégeon F, Delliaux S. The oxidative stress in response to routine incremental cycling exercise in healthy sedentary subjects. Resp Physiol Neurobiol. 2004;144(1):81-90.

23. Phillips CM, Dillon CB, Perry IJ. Does replacing sedentary behaviour with light or moderate to vigorous physical activity modulate inflammatory status in adults?. Int J Behav Nutr Phys Act. 2017;14(1):138

24. McClean CM, Clegg M, Shafat $A$, Murphy MH, Trinick T, Duly E, et al. The impact of acute moderate intensity exercise on arterial regional stiffness, lipid peroxidation and antioxidant status in healthy males. Res Sports Med. 2010;19(1):1-13.

25. Lovlin R, Cottle W, Pyke I, Kavanagh M, Belcastro AN. Are indices of free 
radical damage related to exercise intensity?. Euro J Appl Physiol Occu Physiol. 1987;56(3):313-6.

26. Lu K, Wang L, Wang C, Yang Y, Hu D, Ding R. Effects of high-intensity interval versus continuous moderate-intensity aerobic exercise on apoptosis, oxidative stress and metabolism of the infarcted myocardium in a rat model. Molecul Med Reports. 2015;12(2):2374-82.

27. Mendham AE, Donges CE, Liberts EA, Duffield R. Effects of mode and intensity on the acute exercise-induced IL-6 and CRP responses in a sedentary, overweight population. Euro J Appl Physiol. 2011;111(6):1035-45.

28. DeSouza DC, Matos VAF, DosSantos VOA, Medeiros IF, Marinho CSR, Nascimento PRP, et al. Effects of High-Intensity Interval and Moderate-Intensity Continuous Exercise on Inflammatory, Leptin, IgA and Lipid Peroxidation Responses in Obese Males. Front Physiol. 2018;9:567.

29. Cotman CW, Berchtold NC. Exercise: A behavioral intervention to enhance brain health and plasticity. Tren Neurosci. 2002;25(6):295-301.

30. Swain RA, Harris AB, Wiener EC, Dutka MV, Morris HD, Theien BE, et al.
Prolonged exercise induces angiogenesis and increases cerebral blood volume in primary motor cortex of the rat. Neuroscience. 2003;117(4):1037-46.

31. Stroth S, Kubesch S, Dieterle K, Ruchsow M, Heim R, Kiefer M. Physical fitness, but not acute exercise modulates event-related potential indices for executive control in healthy adolescents. Brain Res. 2009;1269:114-24.

32. Barella LA, Etnier JL, Chang YK. The immediate and delayed effects of an acute bout of exercise on cognitive performance of healthy older adults. J Aging Phys Act. 2010;18(1):87-98.

33. Den BV, Saliasi E, DeGroot RH, Jolles J, Chinapaw MJ, Singh AS. Physical activity in the school setting: Cognitive performance is not affected by three different types of acute exercise. Front Psychol. 2016;7:723.

34. Hillman $\mathrm{CH}$, Snook EM, Jerome GJ. Acute cardiovascular exercise and executive control function. Int J Psychophysiol. 2003;48(3):307-14.

35. Antunes HK, Santos RF, Cassilhas R, Santos RV, Bueno OF, DeMello MT. Reviewing on physical exercise and the cognitive function. Rev Bras Med Esporte. 2006;12(2):108-14.

Cite this article: Gowsi K, Pal GK, Subramanian K. Comparison of Acute Bout of Moderate Intensity and High Intensity Exercise on Oxidative Stress, Inflammatory Markers and Neurocognitive Parameters between Male Athletes and Non-athletes - A Randomized Controlled Study. Int J Clin Exp Physiol. 2019;6(4):139-43. 